\title{
Analysis of clamping versus cutting of T3 sympathetic nerve for severe palmar hyperhidrosis
}

\author{
Ted K. Yanagihara, MPhil, ${ }^{\mathrm{a}}$ Ali Ibrahimiye, MD, ${ }^{\mathrm{b}}$ Catherine Harris, MD, ${ }^{\mathrm{c}}$ Joy Hirsch, PhD, ${ }^{\mathrm{d}}$ and \\ Lyall A. Gorenstein, $\mathrm{MD}^{\mathrm{b}}$
}

\begin{abstract}
Objectives: Endoscopic thoracic sympathectomy can predictably eliminate the disabling symptoms of palmar hyperhidrosis. Debate has ensued over competing techniques, in particular, cutting versus clamping of the sympathetic chain. We subjectively assessed the sweat severity in different areas of the body and evaluated changes in the quality of life in patients undergoing either the cutting or clamping technique.
\end{abstract}

\begin{abstract}
Methods: Patients examined between June 2003 and March 2007 were asked to quantify the severity of their symptoms before and after endoscopic thoracic sympathectomy. The interviews were conducted approximately 1 year after the procedure. Only the patients undergoing sympathectomy at the T3 level for a chief complaint of palmar hyperhidrosis were included in the analysis $(n=152)$. In $45 \%$ of these patients, clamping of the sympathetic chain was performed, and the remaining $55 \%$ had the chain cut.
\end{abstract}

Results: After surgery, no patients had continued excessive sweating of the hands. Of all the patients, $95 \%$ were satisfied with the results after the cutting procedure and $97 \%$ were satisfied after clamping. No difference was seen in any outcome between the patients undergoing clamping versus cutting of the sympathetic chain, including sweating on the hands, face, armpits, feet, trunk, and thighs or in the quality of life.

Conclusions: We found high rates of success and patient satisfaction when T3 sympathectomy was performed for palmar hyperhidrosis, with no differences found between the cutting and clamping techniques. ( $\mathrm{J}$ Thorac Cardiovasc Surg 2010;140:984-9)

Approximately $1 \%$ of whites ${ }^{1}$ are affected by chronic palmar hyperhidrosis that is unprovoked by any mental or physical stressor and is greatly exacerbated by anxiety. Furthermore, the effects of profuse hand sweating on patients' professional and social lives cause significant decrement to their quality of life (QOL). ${ }^{2}$ Despite the current lack of understanding of the etiology or pathophysiology of hyperhidrosis, several methods for surgical sympathectomy have been used to treat patients with severe palmar hyperhidrosis. $^{3}$ The most important factor influencing patient satisfaction and improvement in QOL using any of these procedures has been the appearance of postoperative compensatory sweating (CS). Occasionally, the CS is so severe that it causes a significant decrease in QOL, and patients

From the Department of Neuroscience, ${ }^{\text {a }}$ Medical Scientist Training Program, Columbia University, and the Department of Surgery, ${ }^{b}$ Columbia University College of Physicians and Surgeons, New York, NY; the Department of Surgery, ${ }^{\mathrm{c}}$ University of California, San Francisco, School of Medicine, San Francisco, Calif; and the Program for Imaging and Cognitive Sciences, Departments of Radiology, Psychology, and Neuroscience, ${ }^{\mathrm{d}}$ Columbia University, New York, NY.

Disclosures: None.

Read at the 90th Annual Meeting of The American Association for Thoracic Surgery, Toronto, Ontario, Canada, May 1-5, 2010.

Received for publication May 3, 2010; revisions received June 25, 2010; accepted for publication Aug 9, 2010.

Address for reprints: Ted K. Yanagihara, MPhil, 710 W 168th St, Room B41, New York, NY 10032 (E-mail: tky2102@columbia.edu).

0022-5223/\$0.00

Published by Elsevier Inc. on behalf of The American Association for Thoracic Surgery

doi:10.1016/j.jtcvs.2010.08.014 might regret having undergone endoscopic thoracic sympathectomy (ETS) and wish to have it reversed. No clinical parameters are available that accurately predict which patients will experience severe CS.

Recent studies have examined how variations in the method of sympathectomy can alter the results ${ }^{4,5}$; however, no agreement has been reached regarding the method of ETS. Sympathectomy at the T2 level or at multiple levels has been associated with a greater incidence of severe CS, and consensus has been growing that limiting the extent of sympathectomy will maximize patient satisfaction and minimize the risk of severe CS. ${ }^{6,7}$ The surgical technique has also varied in the method of sympathectomy, and the debate has continued concerning the risks and benefits of cutting versus clamping the nerve. Cutting the sympathetic nerve ensures relief of palmar hyperhidrosis; however, this precludes the potential for reversal in the event of severe CS. An alternative is to place surgical clips on the sympathetic chain, which immediately interrupts the nerve conduction, but can be removed if the patient is unsatisfied with the results. Not all patients who undergo clamp removal will experience a reversal of CS. Several studies have shown good results with clamping at various levels, ${ }^{8-10}$ and one study contrasted the effects of cutting and clamping at multiple thoracic levels. ${ }^{11}$ However, no single study has directly compared the long-term effects of cutting versus clamping when the sympathectomy has been restricted to T3.

Given these factors, an essential need exists for comparisons of the efficacy, incidence of CS, effects on QOL, and patient 


\section{Abbreviations and Acronyms}

$\mathrm{CS}=$ compensatory sweating

ETS = endoscopic thoracic sympathectomy

HDSS $=$ hyperhidrosis disease severity scale

$\mathrm{QOL}=$ quality of life

satisfaction both across and within studies of 1 or more variations to the surgical technique. We administered pre- and postoperative questionnaires regarding the subjectively assessed sweat production, QOL, and overall satisfaction to a cohort of 152 patients with severe palmar hyperhidrosis who underwent T3 ETS either by cutting or clamping.

\section{MATERIALS AND METHODS}

The local institutional review board approved the present study. Between June 2003 and March 2007, 152 patients (82 females; median age, 25 years; range, $12-56$ years) with severe palmar hyperhidrosis underwent T3 ETS at New York Presbyterian Hospital. The baseline subjective sweat characteristics are listed in Table 1. From June 2003 to February 2005, all patients underwent the $\mathrm{T} 3$ cutting procedure; however, our technique then changed to sympathetic nerve clamping, and this technique was performed on all patients until the end of our study period. The surgical technique was identical in all patients. In those undergoing clamping, a 5-mm hemoclip was placed above and below the third ganglion in the same location as the nerve was cut in those patients for whom cutting was performed.

Evaluations were performed using the hyperhidrosis disease severity scale (HDSS). ${ }^{12,13}$ All patients completed the HDSS and a separate QOL evaluation preoperatively. A postoperative surgical questionnaire was completed by all patients (median, 488 days from surgery; range, 1721159 days) and included the HDSS, QOL evaluation, and overall patient satisfaction. The QOL assessment was made from an adapted version of the Liebowitz Disability Self-Rating Scale. ${ }^{14}$ The HDSS uses a scale of 1 to 4 , with $1=$ none, $2=$ mild, $3=$ moderate, and $4=$ severe. The patients were asked to rate the sweating on their face, hands, feet, armpits, trunk, and thighs. Using the same scale, the patients reported how greatly hyperhidrosis had affected their QOL, with 1 indicating a significant decrease and 4 indicating no effect on QOL. Additionally, the preoperative interview measured the baseline characteristics and included a history of medical treatment of hyperhidrosis. The patients were considered to have CS in a body region if an increase in sweating had occurred of 1 point on the HDSS for that area.

Our surgical technique used two 5-mm ports. A 5-mm hemoclip was placed across the interganglionic segment of the sympathetic trunk, immediately inferior to the third and fourth ribs. In patients in whom the nerve was cut, the sympathetic chain was divided immediately below the third and fourth nerve in the exact location at which the nerve in the other group was clamped.

When measuring the effects of surgery, while pooling across the clamping and cutting procedures, the $P$ values were derived using a 2-tailed Wilcoxon signed rank test. The tests for differences between cutting and clamping were performed using the nonparametric Wilcoxon rank sum test. No corrections were made for multiple testing. All data were analyzed using the Statistical Analysis System software (SAS Institute Inc, Cary, $\mathrm{NC})$.

\section{RESULTS}

No deaths, major surgical complications, or occurrences of Horner syndrome occurred. Of the 152 patients who
TABLE 1. Endoscopic thoracic sympathectomy group characteristics

\begin{tabular}{|c|c|c|c|}
\hline HDSS & Pre-ETS & After clamping & After cutting \\
\hline \multicolumn{4}{|l|}{ Hands } \\
\hline None (1) & $0(0)$ & $0(0)$ & $0(0)$ \\
\hline Mild (2) & $1(0.7)$ & $0(0)$ & $1(1)$ \\
\hline Moderate (3) & $12(8.3)$ & $9(14.3)$ & $3(4)$ \\
\hline Severe (4) & $126(91)$ & $54(85.7)$ & $72(95)$ \\
\hline \multicolumn{4}{|l|}{ Face } \\
\hline None (1) & $52(39.7)$ & $21(34)$ & $31(45)$ \\
\hline Mild (2) & $58(42.3)$ & $28(45)$ & $30(43)$ \\
\hline Moderate (3) & 15 (11.5) & $8(13)$ & $7(10)$ \\
\hline Severe (4) & $6(4.6)$ & $5(8)$ & $1(1)$ \\
\hline \multicolumn{4}{|l|}{ Blushing } \\
\hline None (1) & $48(41.4)$ & $26(42)$ & $22(41)$ \\
\hline Mild (2) & 46 (39.7) & $22(35)$ & $24(44)$ \\
\hline Moderate (3) & $19(16.4)$ & $12(19)$ & 7 (13) \\
\hline Severe (4) & $3(2.6)$ & $2(3)$ & $1(2)$ \\
\hline \multicolumn{4}{|l|}{ Armpits } \\
\hline None (1) & $14(10)$ & $5(8)$ & $9(12)$ \\
\hline Mild (2) & $44(33)$ & $23(38)$ & $21(28)$ \\
\hline Moderate (3) & $31(23)$ & $14(23)$ & $17(22.7)$ \\
\hline Severe (4) & $46(34)$ & $18(30)$ & $28(37.3)$ \\
\hline \multicolumn{4}{|l|}{ Feet } \\
\hline None (1) & $3(2.1)$ & $0(0)$ & $3(4)$ \\
\hline Mild (2) & $10(7)$ & $4(6)$ & $6(8)$ \\
\hline Moderate (3) & $34(25)$ & $20(32)$ & $14(19)$ \\
\hline Severe (4) & $90(66)$ & $39(62)$ & $51(69)$ \\
\hline \multicolumn{4}{|l|}{ Trunk } \\
\hline None (1) & $62(47.7)$ & $25(40)$ & $37(54)$ \\
\hline Mild (2) & $56(43.1)$ & $32(52)$ & $24(35)$ \\
\hline Moderate (3) & $11(8.5)$ & $4(6)$ & $7(10)$ \\
\hline Severe (4) & $1(0.8)$ & $1(2)$ & $0(0)$ \\
\hline \multicolumn{4}{|l|}{ Thighs } \\
\hline None (1) & $54(50.9)$ & $30(48)$ & $24(55)$ \\
\hline Mild (2) & $46(43.4)$ & $28(45)$ & $18(41)$ \\
\hline Moderate (3) & $5(4.7)$ & $3(5)$ & $2(5)$ \\
\hline Severe (4) & $1(0.9)$ & $1(2)$ & $0(0)$ \\
\hline \multicolumn{4}{|l|}{ QOL (score) } \\
\hline Not affected (1) & $0(0)$ & $0(0)$ & $0(0)$ \\
\hline Mildly affected (2) & $3(2.1)$ & $1(2)$ & $2(3)$ \\
\hline Moderately affected (3) & $29(20.9)$ & $18(29)$ & $11(14)$ \\
\hline Severely affected (4) & $107(77)$ & $44(70)$ & $63(83)$ \\
\hline
\end{tabular}

Data are presented as number (\%). HDSS, Hyperhidrosis disease severity scale; ETS, endoscopic thoracic sympathectomy; $Q O L$, quality of life.

completed both the pre- and the postoperative questionnaires within the accepted dates, some chose not to answer particular questions. These were not included in the number of responders in the present analyses. In $100 \%$ of patients, ETS decreased the severity of hand sweating, and in $93 \%$, it increased the QOL (Figure 1). A mixture of increases, decreases, and no changes in sweating or facial blushing was evident for other regions. Although not the indication for surgery, $63 \%$ of patients reported a decrease in plantar sweating, $60 \%$ reported a decrease in axillary sweating, and $35 \%$ experienced a decrease in facial blushing. On 


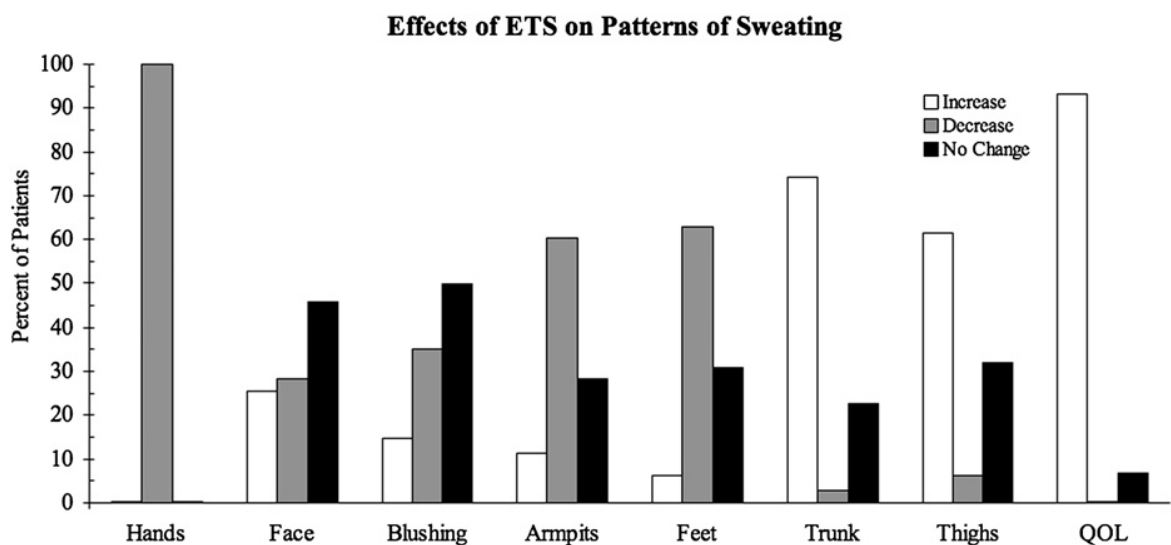

FIGURE 1. Patients were asked to judge the severity of sweating, severity of facial blushing, and quality of life (QOL) at the initial interview and again approximately 1 year postoperatively. The difference between these scores was used as an index of the change, or lack, in symptoms or QOL. For all body regions, except the hands, variable effects of surgery occurred—some patients experienced increases, some decreases, and others had no change in symptom severity.

the thighs and trunk, $61 \%$ and $74 \%$, respectively, reported an increase in sweating.

Data from the postoperative questionnaire from these patients is listed in Table 2, in which patients were stratified by the technique used (cutting or clamping). As expected, ETS had the most pronounced effect on hand sweating, quantified as a subjective reduction in hand sweating. However, most importantly, no significant difference was detected between the clamping and cutting procedures. All patients had

TABLE 2. Quantified effects of ETS on subjective sweat severity

\begin{tabular}{|c|c|c|c|c|}
\hline & All patients & Clamping & Cutting & n $(P$ value $)$ \\
\hline \multicolumn{5}{|l|}{ Hands } \\
\hline Pre-ETS & 3.90 & 2.85 & 2.93 & \\
\hline Post-ETS & 1.25 & 0.14 & 0.35 & \\
\hline Difference & -2.64 & -2.71 & -2.57 & $145(.441)$ \\
\hline \multicolumn{5}{|l|}{ Armpits } \\
\hline Pre-ETS & 2.79 & 1.75 & 1.82 & \\
\hline Post-ETS & 1.88 & 0.86 & 0.87 & \\
\hline Difference & -0.92 & -0.88 & -0.95 & $146(.566)$ \\
\hline \multicolumn{5}{|l|}{ Feet } \\
\hline Pre-ETS & 3.53 & 1.69 & 1.78 & \\
\hline Post-ETS & 2.75 & 2.55 & 2.52 & \\
\hline Difference & -0.78 & -0.85 & -0.74 & $143(.443)$ \\
\hline \multicolumn{5}{|l|}{ Trunk } \\
\hline Pre-ETS & 1.61 & 1.73 & 1.60 & \\
\hline Post-ETS & 2.69 & 0.69 & 0.55 & \\
\hline Difference & 1.08 & 1.03 & 1.04 & $136(.692)$ \\
\hline \multicolumn{5}{|l|}{ Thighs } \\
\hline Pre-ETS & 1.55 & 1.30 & 1.30 & \\
\hline Post-ETS & 2.33 & 0.59 & 0.5 & \\
\hline Difference & 0.78 & 0.70 & 0.80 & $112(.178)$ \\
\hline \multicolumn{5}{|l|}{ QOL } \\
\hline Pre-ETS & 1.64 & 0.65 & 0.62 & \\
\hline Post-ETS & 3.74 & 2.68 & 2.80 & \\
\hline Difference & 2.10 & 2.03 & 2.17 & $145(.627)$ \\
\hline
\end{tabular}

Data are presented as mean values. Abbreviations as in Table 1. reported that hyperhidrosis impaired their QOL at baseline, and most reported that the effect was severe (Table 1). After surgery, the patients in the clamping and cutting groups both reported greatly improved QOL. Again, no significant differences were observed between the 2 groups.

Next, we sought to identify differences in sweating after either cutting or clamping of the sympathetic chain for other body regions and for patient satisfaction (Table 3). The data indicated no significant differences between cutting and clamping across all parameters, including patient satisfaction (Tables 2 and 3). Our final analysis confirmed the presence of CS, with an incidence comparable between the cutting and clamping groups across all body regions (Table 4).

\section{DISCUSSION}

In the presented analysis, we quantified the changes in the patterns of sweating approximately 1.5 years after ETS for palmer hyperhidrosis and found highly favorable results for nearly all 152 patients. Our results support T3 ETS as an

TABLE 3. Post-ETS questionnaire: Comparison of cutting and clamping

\begin{tabular}{|c|c|c|c|}
\hline & $\begin{array}{c}\text { Cutting } \\
(\%)\end{array}$ & $\begin{array}{c}\text { Clamping } \\
(\%)\end{array}$ & $\begin{array}{c}P \\
\text { value }\end{array}$ \\
\hline \multicolumn{4}{|c|}{ Are you satisfied with the results of surgery? } \\
\hline Yes & 95 & 97 & .741 \\
\hline \multicolumn{4}{|c|}{$\begin{array}{l}\text { Have you experienced any new sweating } \\
\text { since surgery? }\end{array}$} \\
\hline Yes & 62 & 70 & .365 \\
\hline \multicolumn{4}{|c|}{$\begin{array}{l}\text { Would you recommend the procedure to } \\
\text { family? }\end{array}$} \\
\hline Yes & 96 & 94 & .619 \\
\hline \multicolumn{4}{|c|}{$\begin{array}{l}\text { Based on the results, would you do the } \\
\text { procedure again? }\end{array}$} \\
\hline Yes & 96 & 94 & .394 \\
\hline
\end{tabular}


TABLE 4. Increased sweating after ETS stratified by technique

\begin{tabular}{lccc}
\hline Region & Cutting (n) & Clamping (n) & $\boldsymbol{P}$ value \\
\hline Face & $20(29 \%)$ & $13(21 \%)$ & .05 \\
Feet & $4(5 \%)$ & $5(8 \%)$ & .06 \\
Armpits & $9(12 \%)$ & $5(8 \%)$ & .35 \\
Trunk & $50(74 \%)$ & $45(73 \%)$ & .24 \\
Thighs & $33(75 \%)$ & $33(53 \%)$ & .14 \\
Blushing & $10(19 \%)$ & $7(11 \%)$ & .85 \\
\hline
\end{tabular}

effective treatment of intractable palmar hyperhidrosis, with a high rate of patient satisfaction and minimal side effects. Maximizing patient satisfaction requires a balance between the relief of palmar sweating and the emergence of CS. It is well established that the development of severe CS negatively affects patient satisfaction. Despite finding CS in at least 1 part of the body in $87 \%$ of our patients, with $4 \%$ reporting severe CS, $97 \%$ were satisfied with the results of surgery.

Our analysis identified no preoperative marker to predict the appearance or severity of CS in either treatment group. The severity of preoperative sweating on the thighs or trunk was not a reliable predictor of developing severe CS or an improvement in QOL. Currently, no method is available to stratify the patients into groups that would predict the risk of severe CS, coincidental decreases in plantar or axillary sweating, and so forth. However, studies quantifying the outcomes of sympathectomy will not only engender more informed physicians and patients, but might also lead to the emergence of predictors of the postoperative results. Several lines of research are evaluating ETS and developing hypotheses about the relationship between the surgery and its outcomes; however, the debate over their interpretations is ongoing.

Several hypotheses have been presented to explain the appearance of CS. Chou and colleagues ${ }^{8}$ have proposed a mechanism for CS in which higher sympathetic blockades (i.e., above T3) disrupt a large number of afferent inhibitory fibers that normally function to dampen hypothalamic sudomotor output. Under this hypothesis, increased rates of CS would depend on the level of cutting or clamping and not on the extent of denervation. A contending proposal has posited that it is the location of symptoms (i.e., facial hyperhidrosis/blushing) and not the level of sympathectomy (i.e., T2$\mathrm{T} 3$ vs T3 alone) that influences the degree of CS. ${ }^{5,15}$ It is possible that both views are partially correct and that both the patient group and surgical technique used are 2 among many factors that influence the surgical outcomes. With consideration of these issues, the cause of CS is not known and accurate hypotheses to explain this phenomenon are difficult to form without an understanding of the neural basis for palmar hyperhidrosis.

Our cohort permitted an opportunity to compare 2 methods of sympathectomy, cutting and clamping of the sympathetic chain at the T3 level. Our motivation for using the clamping technique was the potential to remove the clips in a second surgery if the patient was dissatisfied with the results. Removing clips from the sympathetic chain has been shown to reverse the results of the initial surgery in approximately $50 \%$ of cases when performed promptly. ${ }^{16}$ However, it must be noted that no formal study of the reversal surgery has been performed, and these results must be interpreted with caution. Many surgeons prefer full ablation or cutting of the sympathetic nerve to ensure interruption of the sudomotor signals that lead to palmar sweating. However, ablation or cutting is done at the expense of having the potential to reverse the surgery in the event that severe CS results. A disadvantage of the clamping method is the potential loss of efficacy over time. However, our data indicated no significant differences in any of our measures, including the decrease in hand sweating, the appearance of CS, patient satisfaction, or the effect of ETS on QOL between cutting and clamping.

A limitation of the present study that should be considered was the availability of a large group of patients who had undergone reversal of the clamping surgery. Data from these patients would be highly valuable when informing patients regarding the success rates of reversal. However, because of the rare incidence of such cases, this is difficult to quantify. From the findings we have presented, we recommend the use of surgical clamps restricted to T3 for palmar hyperhidrosis and call for additional studies to replicate our results in larger patient groups.

\section{References}

1. Adar R, Kurchin A, Zweig A, Mozes M. Palmar hyperhidrosis and its surgical treatment: A report of 100 cases. Ann Surg. 1977;186:34-41.

2. Milanez de Campos JR, Kauffman P, de Campos Werebe E, Oliveira Andrade Filho L, Kusniek S, Wolosker N, et al. Quality of life, before and after thoracic sympathectomy: Report on 378 operated patients. Ann Thorac Surg. 2003;76:886-91.

3. Zackrisson T, Eriksson B, Hosseini N, Johnels B, Krogstad AL. Patients with hyperhidrosis have changed grip force, coefficient of friction and safety margin. Acta Neurol Scand. 2008;117:279-84.

4. Ro K, Cantor RM, Lange KL, Ahn SS. Palmar hyperhidrosis: Evidence of genetic transmission. J Vasc Surg. 2002;35:382-6.

5. Baumgartner F, Konecny J. Compensatory hyperhidrosis after sympathectomy: Level of resection versus location of hyperhidrosis. Ann Thorac Surg. 2007;84 1422 .

6. Dewey TM, Herbert MA, Hill SL, Prince SL, Mack MJ. One-year follow-up after thoracoscopic sympathectomy for hyperhidrosis: Outcomes and consequences. Ann Thorac Surg. 2005;81:1227-33.

7. Miller DL, Bryant AS, Force SD, Miller JI. Effect of sympathectomy level on the incidence of compensatory hyperhidrosis after sympathectomy for palmar hyperhidrosis. J Thorac Cardiac Surg. 2009;138:581-5.

8. Chou SH, Kao EL, Lin CC, Chang YT, Huang MF. The importance of classification in sympathetic surgery and a proposed mechanism for compensatory hyperhidrosis: Experience with 464 cases. Surg Endosc. 2006;20:1749-53.

9. Lin CC, Mo LR, Lee LS, Ng SM, Hwang MH. Thoracoscopic T2-sympathetic block by clipping-A better and reversible operation for treatment of hyperhidrosis palmaris: Experience with 326 cases. Eur J Surg. 1998;580(Suppl):13-6.

10. Lin TS, Chou MC. Treatment of palmar hyperhidrosis using needlescopic T2 sympathetic block by clipping: Analysis of 102 cases. Int Surg. 2004;89:198-201.

11. Reisfeld RR, Nguyen R, Pnini A. Endoscopic thoracic sympathectomy for hyperhidrosis: Experience with both cauterization and clamping methods. Surg Lap Endo Perc Tech. 2002;12:255-67. 
12. Kowalski JW, Eadie N, Daggett S, Lai PY, Mordaunt J, Strutton D, et al. Validity and reliability of the hyperhidrosis disease severity scale (HDSS). Poster presented at 62nd Annual Meeting of the American Academy of Dermatology, February 2004, Washington DC; poster P198.

13. Lowe NJ, Glaser DA, Eadie N, Daggett S, Kowalski JW, Lai PY. Botulinum toxin type A in the treatment of primary axillary hyperhidrosis: A 52-week multicenter double-blind randomized, placebo-controlled study of efficacy and safety. $J \mathrm{Am}$ Acad Dermatol. 2007;56:604-11.

14. Schneier FR, Heckelman LR, Garfinkel R, Campeas R, Fallon BA, Gitow A, et al. Functional impairment in social phobia. J Clin Psych. 1994;55:322-31.

15. Baumgartner FJ, Toh Y. Severe hyperhidrosis: Clinical features and current thoracoscopic surgical management. Ann Thorac Surg. 2003;76:1878-83.

16. Kang CW, Choi SY, Moon SW, Cho DG, Kwon JB, Sim SB, et al. Short-term and intermediate-term results after unclipping: What happened to primary hyperhidrosis and truncal reflex sweating after unclipping in patients who underwent endoscopic thoracic sympathetic clamping? Surg Lap Endo Perc Tech. 2008;18:469-73.

\section{Discussion}

Dr M. Blair Marshall (Washington, DC). Thank you for an excellent presentation and for getting me both your talk and your manuscript in advance. In discussing sympathectomy, different groups do it different ways. You state that it is a T3 sympathectomy, but you clipped or cut at 2 levels. Could you clarify the definition of what you think T3 is or what one would consider a T3 and T4 sympathectomy?

Dr Ibrahimiye. In our method, we clip immediately inferior to the third and the fourth ribs, isolating the third interganglionic segment of the sympathetic trunk. I think in other studies, they used the term "T3-T4 sympathectomy" for the procedure that we did.

Dr Marshall. This was a retrospective review. It was unclear to me how many patients responded to your survey. Did you have $100 \%$ follow-up? Was it every patient followed up exactly at 1 year after their sympathectomy or was the survey sent out at one point for all patients?

Dr Ibrahimiye. The survey was sent out to all patients at one point, but the patients responded at different intervals. Also, the follow-up was $100 \%$, but not all patients responded to all the questions. You might have seen in the manuscript that for different parts of the body, especially postoperatively, the patients chose not to answer some questions. That is the reason for the discrepancy in the manuscript. We only included the patients who responded.

Dr Marshall. In your survey you describe trunk or thigh sweating, but you did not differentiate between the groin or behind the knees, which can be problematic for many patients. Do you think that patients understand the meaning of trunk sweating, and what do you do if you have horrific groin sweating? How do you respond to your questionnaire?

Dr Shrager. You probably do not know whether patients knew what you meant by trunk. I do not know how he could answer that.

Dr Marshall. I have found when I discuss compensatory sweating (CS) with patients, if you use the word "trunk," they do not really know what you are talking about. So how do you ensure, in fact, that the patients know enough to answer your survey appropriately?

Dr Ibrahimiye. I think you make a very valid point. Obviously, when we say "trunk," we mean the torso, but some patients, as you pointed out, might not understand what we mean, and I do not believe we made an effort to tell them what we mean by "trunk."
Dr Mark J. Krasna (Towson, Md). Ali, thank you. It is actually a very intriguing paper.

I want to follow-up on one of Dr Marshall's points, which I think is very important. There is actually a new standard nomenclature for reporting sympathectomy that has been adopted by both the Society of Thoracic Surgeons and the International Society of Sympathetic Surgery. I would strongly recommend before your final version of your manuscript that you go to one of those Web sites and you will see that. I think what Blair was pointing out is that what you really did is what would be called an R3-R4 sympathetic clamping, and that is probably how it should be defined.

I do have 2 questions, though, which I think are very intriguing to all of us who have been looking at the question of clamping. One very simple question is did you notice a difference in the operating time when you did clamping versus cutting? You did not tell us, but did you look for it?

Dr Ibrahimiye. We did not look for it specifically, but this was a single-surgeon, single-institution experience, and they reported that there was no significant time difference.

Dr Krasna. My second question has to do with reversibility. Obviously, one of the benefits proposed for doing clamping versus cutting is, as you said, the potential for reversibility. Did you actually have any cases in whom the patients were unhappy and they went for reversibility, and if those patients underwent a reversal procedure, was it successful? That is obviously one of the key questions we would like to know.

Dr Ibrahimiye. Thank you.

In this cohort of 152 patients, we actually had 1 patient who underwent a reversal procedure 1 year after the original procedure, and this patient did not have any improvement of her symptoms. It was 1 year after the initial procedure. In addition to that, in our entire cohort of 362 patients, we had 4 additional patients who underwent a reversal procedure. Three of these four actually reported improvement and were able to maintain the benefits of the original procedure; however, one did not have any change in symptoms, and the patient who did not have any change was also 1 year out of the surgery.

Dr Laureano Molins (Barcelona, Spain). Thank you for your presentation.

Our experience was with cutting until 2007, 300 patients, and then 100 more with clipping, and the results were quite similar. I have 2 questions. One is technical. What kind of clip do you use? We think that the usual surgical clip, you cannot see the end. Second, with these conclusions, do you recommend, as we are doing, clipping versus cutting?

Dr Ibrahimiye. We use the standard 5-mm hemoclip. As you pointed out, the most important thing that my attending always tells me is that you have to see the ends of the clips, as you pointed out, but it is a straight 5-mm hemoclip.

Your second question, we do recommend this procedure for those patients who have failed medical therapy and in whom hyperhidrosis is severely affecting their quality of life, and we always make them aware of the possibility of compensatory sweating.

Dr Molins. So you have adopted the clipping?

Dr Ibrahimiye. Yes, we have completely adopted the clipping method.

Dr Michael A. Maddaus (Minneapolis, Minn). You indicated that you do a T3 and T4 clipping routinely. Is that right? 
Dr Ibrahimiye. Yes.

Dr Maddaus. Is that for all comers, kind of like a Nissen for all refluxers? In other words, for people who have axillary hyperhidrosis, do you do a T3-T4? You know, when you get the occasional patient with a foot problem, do you do a T3-T4? Do you differentiate in any way what levels you clip, or is everybody a T3-T4?

Dr Ibrahimiye. Everybody in this study cohort had palmar hyperhidrosis, so they all underwent the same procedure. I have not had any experience with axillary hyperhidrosis. Perhaps, Dr Gorenstein might be able to answer your question.

Dr Lyall A. Gorenstein (New York, NY). The only operation we do basically is for patients who have palmar hyperhidrosis. So the axillaries, the plantars, et cetera, they are treated in other ways.

Dr Maddaus. You do not do it for axillary then?

Dr Gorenstein. No.

Dr Maddaus. Anything for the head?

Dr Gorenstein. No.

Dr Maddaus. Any experience with that?
Dr Gorenstein. We did in the past and stopped doing it because of bad outcomes, either disappointed patients or severe compensatory sweating, and do not recommend it.

Dr Joseph B. Shrager (Stanford, Calif). It has become traditional when there is a paper on hyperhidrosis to poll the audience to see what people do.

For isolated palmar hyperhidrosis, who does T4 only?

(A show of hands.)

Dr Shrager. One. Who does T3 only?

(A show of hands.)

Dr Shrager. Who does T3 and T4?

Dr Krasna. R3-R4.

Dr Shrager. Okay. Sorry, Dr Krasna. R3 and R4?

(A show of hands.)

Dr Shrager. Who does R2 through R4?

(A show of hands.)

Dr Shrager. Just R2? Any others just R2?

(A show of hands.)

Dr Shrager. I know you have changed, then, Blair, because that is not what you told me a year ago. 